\title{
A novel brain metastasis xenograft model for convection-enhanced delivery of targeted toxins via a micro-osmotic pump system enabled for real-time bioluminescence imaging
}

\author{
JUN HUANG ${ }^{1}$, YAN MICHAEL LI ${ }^{2}$, QUAN CHENG ${ }^{1}$, DANIEL A. VALLERA $^{3}$ and WALTER A. HALL ${ }^{2}$ \\ ${ }^{1}$ Department of Neurosurgery, Xiangya Hospital of Central South University, Changsha, Hunan 410008, P.R. China; \\ ${ }^{2}$ Department of Neurosurgery, SUNY Upstate Medical University, Syracuse, NY 13210; \\ ${ }^{3}$ Department of Therapeutic Radiology, University of Minnesota Medical School, Minneapolis, MN 55455, USA
}

Received September 23, 2014; Accepted May 19, 2015

DOI: $10.3892 / \mathrm{mmr} .2015 .4111$

\begin{abstract}
Brain metastasis is a common cause of mortality in patients with cancer, and is associated with poor prognosis. There is a current requirement for the identification of relevant brain metastasis tumor models, which may be used to test novel therapeutic agents and delivery systems in pre-clinical studies. The present study aimed to investigate the development of a murine model of brain metastasis, and the application of bioluminescence imaging (BLI) for monitoring tumor growth and response to targeted toxins (TT). A luciferase-modified human brain metastasis cell line was implanted into the caudate-putamen of athymic mice using a stereotactic frame. Tumor growth was monitored by BLI, and tumor volume was calculated from three-dimensional measurements of serial histopathological sections. Histopathological analyses revealed the presence of tumor growth within the caudate-putamen of all of the mice, and BLI was shown to be correlated with tumor volume. To evaluate whether this model would allow the detection of a therapeutic response, mice bearing metastatic brain tumor cell xenografts were treated with TT delivered by convection-enhanced delivery (CED), via a micro-osmotic pump system. The TT-treated groups were submitted to metastatic brain tumor cell experiments, the results of which suggested that TT treatment delayed tumor growth, as determined by BLI monitoring, and significantly extended the survival of the mice. The results of the present study demonstrated the efficacy of a brain metastasis model for CED of TT via a micro-osmotic pump system in athymic mice, in which tumor growth and response to therapy were accurately monitored
\end{abstract}

Correspondence to: Professor Walter A. Hall, Department of Neurosurgery, SUNY Upstate Medical University, 19750 East Adams Street, Syracuse, NY 13210, USA

E-mail: upstatewalter@163.com

Key words: metastatic brain tumors, animal model, bioluminescence, convection-enhanced delivery, targeted toxin by BLI. In conclusion, this model may be well-suited for pre-clinical testing of potential therapeutics for the treatment of patients with metastatic brain tumors.

\section{Introduction}

Brain metastasis is a common cause of mortality in patients with cancer. It is estimated that $20-40 \%$ of patients with systemic cancer develop metastases to the central nervous system (CNS) (1). Brain metastases are associated with a median survival rate of 4-6 months, despite treatment with surgery, radiation therapy, and chemotherapy (2). Targeted toxins (TT) are extremely potent agents that recognize specific antigens located on tumor cells, and cause cytotoxicity following cell entry, via protein synthesis inhibition $(3,4)$. Previous studies have shown that TT may be appropriate for the treatment of patients with intracranial metastatic disease or with radio-resistant tumor histology $(4,5)$. Unfortunately, due to the large size of TT and the presence of the partially intact blood-brain barrier (BBB), these compounds poorly penetrate the CNS, which ultimately influences the therapeutic efficacy of the delivery system $(4,6)$. Convection-enhanced delivery (CED) represents a potential approach for the delivery of TT to the brain, by bypassing the BBB $(6,7)$. Numerous pre-clinical and early clinical studies have demonstrated that CED is a useful modality for regional drug delivery in the treatment of brain tumors (8-10). In order to develop improved treatment strategies and to improve the outcome for brain metastasis, there is a current requirement for the identification of relevant metastatic brain tumor models, which may be used to test novel therapeutic agents and delivery systems in pre-clinical studies. When using intracranial xenograft models, therapeutic efficacy is usually measured by bioluminescence imaging (BLI), which allows for noninvasive, quantitative, and real-time monitoring of tumor growth and response to therapy in small animal models (11-13).

The present study aimed to develop a metastatic brain tumor xenograft model in athymic mice, using a micro-osmotic pump system for CED of TT, which could be monitored by BLI. This model system adapted for BLI may 
prove useful for pre-clinical testing of therapeutics for the treatment of patients with metastatic brain tumors.

\section{Materials and methods}

Cell culture. The PC9-BrM3 non-small cell lung cancer human metastatic brain tumor cell line was obtained from the Memorial Sloan-Kettering Cancer Center (New York, NY, USA) (14). A luciferase reporter gene (Clontech Laboratories, Inc., Mountain View, CA, USA) was inserted, according to a previously described method (11). The cells were cultured in Dulbecco's modified Eagle's medium supplemented with $10 \%$ fetal bovine serum (Thermo Fisher Scientific, Inc., Logan, UT, USA), and 5\% streptomycin (Mediatech, Inc., Manassas, VA, USA). The cells were grown as monolayers and incubated in a humidified $37^{\circ} \mathrm{C}$ atmosphere containing $5 \% \mathrm{CO}_{2}$. As determined by Trypan blue (Thermo Fisher Scientific, Inc.) exclusion, only cells with $>95 \%$ viability were used for subsequent experiments.

Animals. Six week old female athymic mice weighing 17-19 g were purchased from the National Cancer Institute (Hudson, NY, USA). The mice were housed in an animal facility and were maintained in a temperature-controlled and light-controlled environment, with an alternating $12 \mathrm{~h}$ light/dark cycle. All surgeries were performed under sterile conditions and all animal experimental protocols were approved by the animal care and use committee of SUNY Upstate Medical University (Syracuse, NY, USA).

Surgical procedure for implantation of tumor cells. For intracranial tumor injections, the mice were anesthetized with an intraperitoneal injection of ketamine solution $(80 \mathrm{mg} / \mathrm{kg}$; Sigma-Aldrich, St. Louis, MO, USA). The anesthetized mice were then placed into the ear bars of a stereotactic frame (David Kopf Instruments, Tujunga, CA, USA), as previously described (9). The scalp was swabbed with povidone-iodide (PDI, Orangeburg, NY, USA) and a midline incision was made using a scalpel. A burr hole was drilled through the skull $0.5 \mathrm{~mm}$ anterior to the bregma and $2.5 \mathrm{~mm}$ lateral to the midline using a drill (Foredom Electric Co., Bethel, CT, USA). Using the stereotactic frame a $10 \mu$ l Hamilton syringe attached to a 25 gauge needle was used to deliver the tumor cells to a $3.0 \mathrm{~mm}$ depth from the skull surface into the middle of the caudate-putamen (Fig. 1). Following intracranial inoculation with $1.4 \mu 14 \times 10^{5}$ metastatic brain tumor cells over a period of $5 \mathrm{~min}$, the needle was left in place for a further 5 min prior to being slowly withdrawn. The burr hole was covered with sterile bone wax (Ethicon, Somerville, NJ, USA), and the incision was closed with surgical glue.

Growth kinetics of intracranial tumor xenografts and determination of intracranial tumor volumes. The brains of 16 mice were injected with $4 \times 10^{5}$ luciferase-modified PC9-BrM3 metastatic brain tumor cells, and intratumoral luciferase activity was monitored by BLI. BLI was performed once every 5 days beginning at day five following tumor cell implantation. Four mice were sacrificed at days $10,20,30$, and 40 following implantation and immediately following BLI. The mice were sacrificed with an overdose of inhaled $\mathrm{CO}_{2}$, and their brains were removed and placed in $4 \%$ formalin immediately afterwards. The formalin-fixed murine brains were subsequently deposited in $20 \%$ sucrose solution at $4^{\circ} \mathrm{C}$, embedded in paraffin, sectioned at $200 \mu \mathrm{m}$ intervals, and then stained with hematoxylin and eosin (HE; Sigma-Aldrich). The histological analyses of the serial microscopy sections were performed using a Leica DMR microscope (Leica Microsystems GmbH, Wetzlar, Germany). The digital images of the tumor-containing sections were then examined for each mouse in order to determine the histopathological tumor volumes.

Micro-osmotic pump implantation and treatment of metastatic brain tumors with TT in mice. The osmotic minipump system (ALZET model 1007D; Durect Corporation, Cupertino, CA, USA) consisted of four components: A micro-osmotic pump, a flow moderator, a catheter tube, and a brain infusion cannula (Fig. 2). In order to evaluate the therapeutic response of the metastatic brain tumor xenografts to TT, 12 mice that received the caudate-putamen implantation of luciferase-modified brain metastatic tumor cells were randomly assigned to either TT (DTATEGF) treatment or control groups. The mice were assigned to the groups following the initial BLI image examination, which was performed 4 days following tumor cell implantation. The pump systems were assembled for intracranial infusion according to the manufacturer's instructions, and both a 25 gauge $3 \mathrm{~mm}$ brain infusion needle and a $15 \mathrm{~mm}$ catheter tube were attached to the pump. The drug-loaded pumps were implanted subcutaneously into the mouse back. In the treatment group, each pump reservoir was filled with $1 \mu \mathrm{g}$ TT in $100 \mu 1$ solution. DTATEGF TT is a novel recombinant bispecific TT consisting of a truncated diphtheria toxin (DT), an aminoterminal (AT) fragment of the urokinase-type plasminogen activator, and a fragment of human epidermal growth factor (EGF), and was developed in our laboratory (5). In the control group, the pumps were filled with $100 \mu 1$ BIC3KDEL (University of Minnesota, Minneapolis, MN, USA), a T-cell targeting control toxin. The pump flow rate was set to $0.5 \mu \mathrm{l} / \mathrm{h}$, and the solutions were delivered continuously for 7 days. On day eight following pump implantation, the mice were anesthetized again and all components of the pump system were removed. The mice were monitored each day for the development of symptoms associated with tumor growth and pump implantation. Following tumor cell implantation, the mice were monitored by BLI at days 4, 14, 30,60 , and 90 . The mean BLI values for the treatment and control groups were then calculated and plotted according to the corresponding day of imaging. The mice were sacrificed by $\mathrm{CO}_{2}$ inhalation when they demonstrated physical symptoms indicative of significant compromise to neurological function, or following $>20 \%$ body weight loss.

In vivo BLI of intracranial tumors. In vivo BLI was performed using the IVIS 50 Imaging system (Caliper Life Sciences, Alameda, CA, USA), which captured the luminescence signal emitted from the engrafted tumor. The data were analyzed using Living Image 2.5 software (Caliper Life Sciences). Prior to imaging, the mice were anesthetized with inhalation of isoflurane gas, and injected intraperitoneally with $150 \mathrm{mg} / \mathrm{kg}$ D-luciferin aqueous solution (PerkinElmer, Inc., Waltham, 


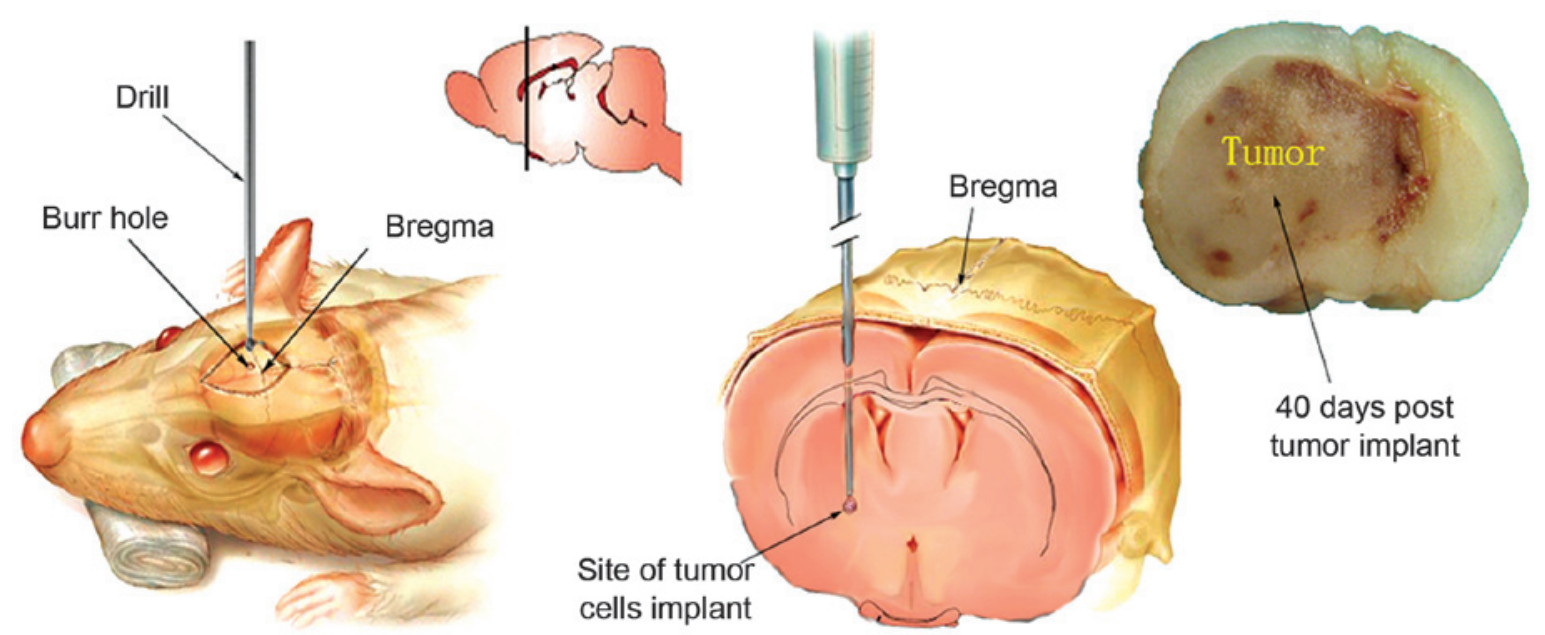

Figure 1. Site of the burr hole and location of tumor cell implantation.

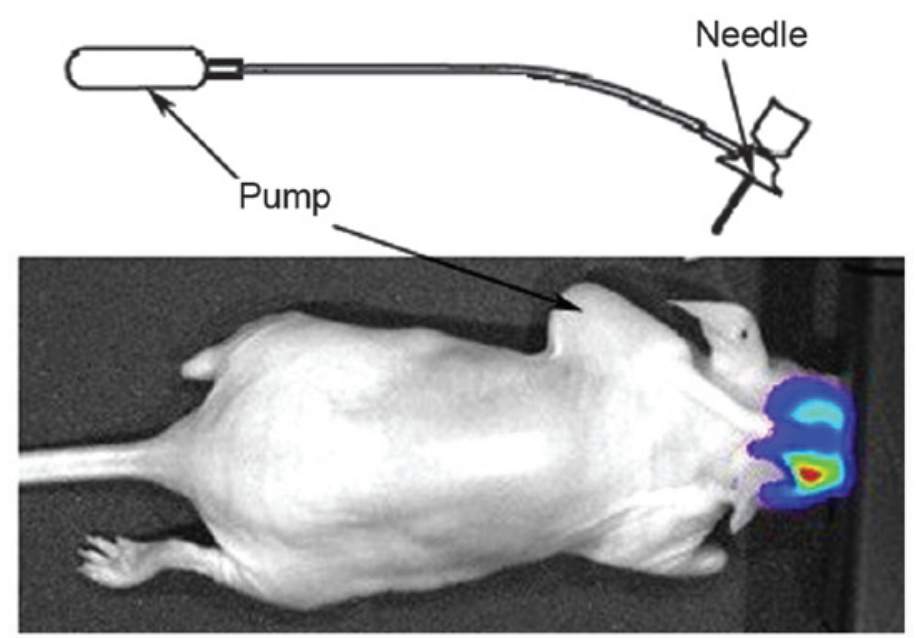

Figure 2. ALZET Brain infusion kit 3 component system and convection-enhanced delivery of targeted toxins for real-time bioluminescence imaging (BLI) in an athymic mouse model. The drug-loaded pump was implanted subcutaneously on the mouse back and the BLI was visualized over its head.

MA, USA). The images were subsequently captured $10 \mathrm{~min}$ following injection. Signal intensity was quantified within a region of interest over the head, as defined by Living Image software. All images represent 2 min exposure time, and the total numbers of photons $/ \mathrm{sec} / \mathrm{cm}^{2} /$ steradian were recorded.

Statistical analysis. The data were statistically analyzed with a Student's t-test using commercially available software (GraphPad Prism; GraphPad Software, Inc. La Jolla, CA, USA). The statistical differences between each comparison were calculated using a log-rank test. The correlation between bioluminescence and corresponding tumor volume was calculated using a linear regression analysis. $\mathrm{P}<0.05$ was considered to indicate a statistically significant difference.

\section{Results}

General outcomes. Injection of luciferase-modified human metastatic brain tumor cells into athymic mice resulted in $100 \%$ tumor growth. During all experimental procedures, no morbidity or mortality was observed in response to the pump system or the surgical procedure. The mice implanted with pumps showed no evidence of clinical side effects over the study period. No mice experienced irritation at the wound site or attempted to remove the pump system. No neurological deficits were observed immediately following cell inoculation, pump implantation, or drug delivery. The pump system never migrated from its implanted position, and all mice tolerated the device well. In the latter stages of the experiments, mild contralateral forelimb weakness was observed and gradually worsened over time. The body weights of the mice began to significantly decrease and the animals deteriorated rapidly in their last week of life prior to being sacrificed, as a consequence of progressive tumor growth.

Real-time imaging of xenograft brain metastases growth as measured by BLI. In a time course study involving tumor monitoring by BLI, the mice were injected with luciferase-modified metastatic brain tumor cells, and sacrificed at selected time points following implantation. Bioluminescence was readily detectable on day five following tumor cell implantation, and the normalized luminescence plot showed progressively increasing luminescence until the mice developed neurological 
A

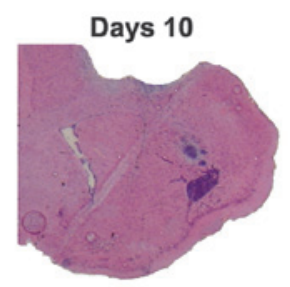

Days 20

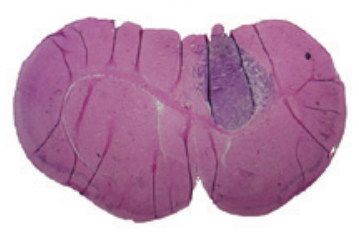

Days 30

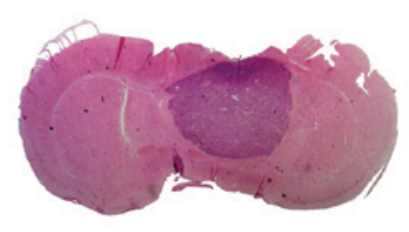

Days 40

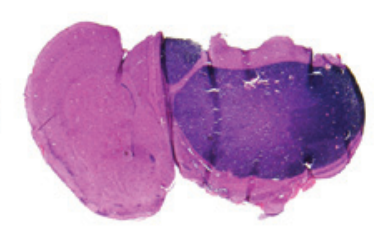

B
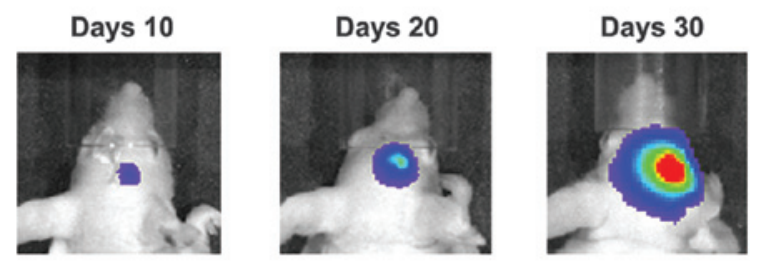

Days 40

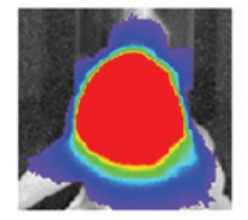

D

C

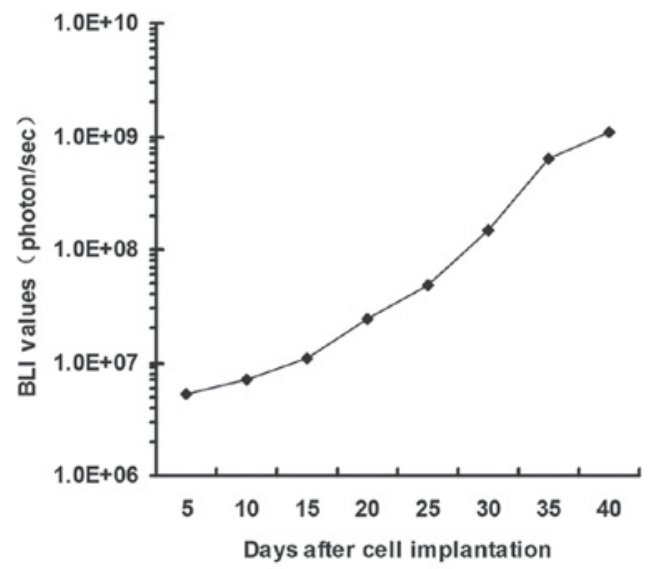

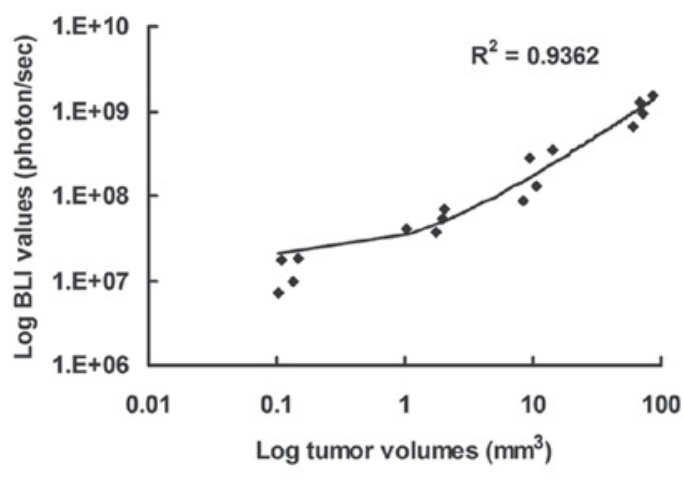

Figure 3. Association between bioluminescence signal and corresponding tumor volume in mice receiving intracerebral injection of metastatic brain tumor cells. A total of 16 mice were injected with PC9-BrM3 luciferase-modified brain metastasis cells, and bioluminescence monitoring was performed 4 days following metastatic brain tumor cell implantation. Four mice were euthanized on days 10, 20, 30, and 40 following bioluminescence imaging (BLI) (A) Histopathological analysis reveals progressive PC9-BrM3 tumor growth from days 10-40 (hematoxylin and eosin, original magnification $\mathrm{x} 40$ ). (B) BLI obtained in the mice prior to euthanization demonstrated a corresponding progressive signal increase from the tumors. (C) BLI shows a corresponding signal increase from the tumors. (D) Linear regression analysis of the correlation between tumor volume and luminescence strength. The correlation coefficient shows a significant association between tumor volume and BLI $\left(\mathrm{R}^{2}=0.94\right)$.

symptoms. Small tumors were detected on day 10 , and tumor size increased up to day 40. The implanted tumor cells exhibited logarithmic growth 10 days following tumor cell implantation. On day 40, the presence of solid tumors was evident in the right caudate-putamen, with extension over the brain surface (Fig. 3A and B).

Correlation between bioluminescence and corresponding tumor volume. The HE-stained sections of each tumor-bearing brain were serially registered and used to estimate tumor volume. The tumor volumes were compared with the corresponding luminescence readings obtained prior to mouse sacrifice (Fig. 3C). Linear regression analyses revealed a marked correlation $\left(\mathrm{R}^{2}=0.94\right)$ between the strength of luminescence and intracerebral tumor volume (Fig. 3C).

TT intracranial metastatic tumor efficacy. BLI monitoring was used to evaluate the therapeutic response of intracranial metastatic brain tumor xenografts. The control group normalized luminescence plot showed a progressive increase in luminescence until the mice developed neurological symptoms such as moribund state, which was used an indicator for

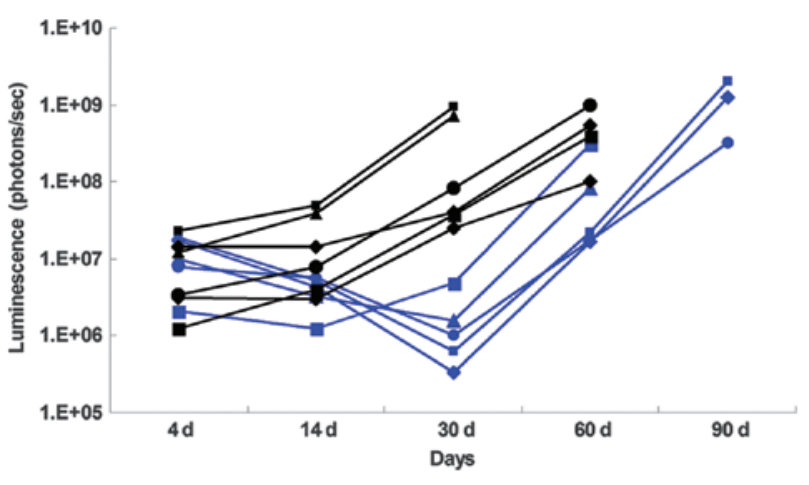

Figure 4. Luminescence levels in the targeted toxin (TT) treatment group, as compared with the control group. In the mice treated with $100 \mu \mathrm{g}$ DTATEGF TT (blue lines), a significant decrease followed by a significant increase in luminescence levels was observed, whereas in the BIC3DEL-treated mice (black lines) a consistent increase in luminescence was observed. Treatment with TT significantly prolonged median murine survival from 63 days in the control group to 87 days in the TT treatment group $(\mathrm{P}=0.006)$.

sacrifice. Luminescence in the TT (DTATEGF)-treated mice was substantially reduced at day 14 when the treatment was completed, and remained low until day 30 following tumor 
cell implantation, as compared with the control group (Fig.4). Survival analyses revealed that the median survival duration of the TT-treated mice was significantly extended, as compared with the control group ( 87 days, vs. 63 days, $\mathrm{P}=0.006$ ).

\section{Discussion}

The systemic delivery of TT to the CNS is restricted due to the inability of numerous compounds to cross the BBB, and the systemic toxicity of TT. Currently, a useful modality for regional drug delivery for brain tumor treatment is $\operatorname{CED}(6,8,10)$. CED is a strategy pioneered by Bobo et al (15) whereby drugs are delivered directly into the tumor or adjacent brain parenchyma via implanted catheters. CED bypasses the BBB and unlike systemic therapy, decreases the potential for systemic toxicity. Previous studies have described the distribution and concentration of chemotherapeutic agents delivered via CED in vivo (16-18).

Previous studies have demonstrated the efficacy of CED-delivered TT $(10,12,13)$. Numerous studies have been performed using CED via a syringe or shunt catheter, where high flow rates are used to infuse large volumes of drug over a short period of time. The results of these studies demonstrated that the infusion may create a deforming force on the underlying tissue, which may in turn cause tearing of the tissue or reflux of the infusate along the catheter track, away from the target tissue (19-22).

In the murine model generated in the present study, CED was performed via a micro-osmotic pump system, to provide a continuous positive-pressure microinfusion, a method that has numerous advantages. Firstly, a drug may be continuously delivered at a constant rate for days without the need for anesthesia or the frequent handling of small animals. In addition, the small, flexible pump system may be fixed to the skull and therefore will not interfere with the normal activities of the mice. The system does not require an external syringe or shunt catheter, thus decreasing the chance for infection. Furthermore, the pump is fully biocompatible and the infusion volume is well tolerated. A DT-based TT delivery by CED was previously shown to exhibit immunological privilege (9). Therefore, the model used in the present study is a simple and effective tool for performing CED treatment experiments in small animals with brain tumors.

Quantitative measurements of therapy-induced changes in tumor growth have been difficult to obtain in animal models, as they require the sacrifice of large numbers of animals at multiple time points. Numerous non-invasive approaches to imaging tumor growth in small animals have become available, including magnetic resonance imaging (MRI), computerized tomography (CT), positron emission tomography (PET), single photon emission computed tomography (SPECT), and ultrasonography (23-25). Each technique has its own advantages and disadvantages. MRI, CT, and ultrasonography produce anatomical images of structures in the body, whereas PET and SPECT image physiological processes and thus produce functional images. These imaging modalities are expensive and require technical expertise. Therefore, the use of two relatively novel alternative non-invasive approaches, fluorescence imaging and BLI, is expanding rapidly $(11,25)$. BLI detects the emission of photons based on energy-dependent reactions catalyzed by cells or organisms that have been genetically modified to express luciferase. BLI is a cost-effective, efficient, and accurate means for assessing tumor growth and response to therapy (11). The applications for BLI have been numerous, and include monitoring tumor cell growth, visualizing tumor regression in response to chemotherapy, determining cell migration, and monitoring the locations as well as the timing of promoter-dependent luciferase expression $(11,12,13,25)$. In the present study, the bioluminescence signal was readily detectable on day 5 following tumor cell implantation, and brain tumor growth could be monitored in real time via BLI. Since this approach involved the direct physical measurement of intracranial tumor for comparison with the BLI results, the extent of the correlation between tumor volume and the strength of the observed luminescence signal was high. With regards to treatment response, the anti-tumor effect of TT was readily detected by BLI, and the effects of the treatment detected by BLI indicated a corresponding increase in animal survival. These results suggested that BLI is a rapid, easy to perform, sensitive, and suitable method to establish early tumor response to treatment, and may be used as an accurate model to assess the effect of therapy on survival. Although this monitoring method has only been validated in experimental models, the results of the present study highlight the potential value of this technique in the rapid evaluation of treatment response in pre-clinical oncology trials. This model may be used in further studies that measure therapeutic index and pharmacokinetics, in order to determine whether other means of drug delivery are superior.

Although the efficacy of a bispecific TT was not the focus of the present study, the results demonstrated that TT could be delivered by CED via a micro-osmotic pump system to the intracranial metastatic brain tumor and delay tumor growth, as indicated by BLI monitoring, and could also significantly extend the survival of the animal subjects. Based on these results, TT is a reasonable treatment alternative that may be used when radiation therapy fails due to systemic toxicity or tumor resistance, and further study regarding this promising agent is warranted.

In conclusion, the present study demonstrated the development of a novel metastatic brain tumor xenograft model for CED of TT via a micro-osmotic pump system that was measured by real-time BLI, which in turn facilitates the pre-clinical testing of novel therapies for the treatment of cancer. The results obtained from the present study indicated that this xenograft model has numerous unique features that may prove useful in the investigation of the pathobiology of intracranial tumor growth, and for monitoring systemic and intracranial responses to anti-tumor agents. Notably, the results demonstrated that TT delivered by CED via a micro-osmotic pump system is an effective drug against metastatic tumor growth, and that BLI accurately defines metastatic tumor growth and is able to demonstrate tumor response to TT. This metastatic brain tumor xenograft model combined with CED and BLI technology may serve as a valuable tool for preclinical screening for drugs effective in targeting metastatic brain tumors.

\section{Acknowledgements}

The present study was supported by the Scientific Research Foundation for Selected Overseas Chinese Scholars, State Education Ministry (2013); and the Technology Foundation 
for Selected Overseas Chinese Scholar, Ministry of Human Resources and Social Security of the People's Republic of China (2014).

\section{References}

1. Arnold SM and Patchell RA: Diagnosis and management of brain metastases. Hematol Oncol Clin North Am 15: 1085-1107, 2001.

2. Gaspar L, Scott C, Rotman M, et al: Recursive partitioning analysis (RPA) of prognostic factors in three Radiation Therapy Oncology Group (RTOG) brain metastases trials. Int J Radiat Oncol Biol Phys 37: 745-751, 1997.

3. Hall WA: Immunotoxin therapy. Neurosurg Clin N Am 7: 537-546, 1996.

4. Rustamzadeh E, Low WC, Vallera DA and Hall WA: Immunotoxin therapy for CNS tumor. J Neurooncol 64: 101-116, 2003.

5. Huang J, Li YM, Massague J, Sicheneder A, Vallera DA and Hall WA: Intracerebral infusion of the bispecific targeted toxin DTATEGF in a mouse xenograft model of a human metastatic non-small cell lung cancer. J Neurooncol 109: 229-238, 2012.

6. Hall WA: Convection-enhanced delivery: Neurosurgical issues. Curr Drug Targets 10: 126-130, 2009.

7. Vogelbaum MA: Convection enhanced delivery for treating brain tumors and selected neurological disorders: Symposium review. J Neurooncol 83: 97-109, 2007.

8. Vogelbaum MA, Sampson JH, Kunwar S, et al: Convection-enhanced delivery of cintredekin besudotox (interleukin-13-PE38QQR) followed by radiation therapy with and without temozolomide in newly diagnosed malignant gliomas: Phase 1 study of final safety results. Neurosurgery 61: 1031-1038, 2007.

9. Oh S, Ohlfest JR, Todhunter DA, et al: Intracranial elimination of human glioblastoma brain tumors in nude rats using the bispecific ligand-directed toxin, DTEGF13 and convection enhanced delivery. J Neurooncol 95: 331-342, 2009.

10. Hall WA, Rustamzadeh E and Asher AL: Convection-enhanced delivery in clinical trials. Neurosurg Focus 14: e2, 2003.

11. Rehemtulla A, Stegman LD, Cardozo SJ, et al: Rapid and quantitative assessment of cancer treatment response using in vivo bioluminescence imaging. Neoplasia 2: 491-495, 2000.

12. Paroo Z, Bollinger RA, Braasch DA, et al: Validating bioluminescence imaging as a high-throughput, quantitative modality for assessing tumor burden. Mol Imaging 3: 117-124, 2004.
13. Szentirmai O,Baker $\mathrm{CH}$, Lin N, et al: Noninvasive bioluminescence imaging of luciferase expressing intracranial U87 xenografts: Correlation with magnetic resonance imaging determined tumor volume and longitudinal use in assessing tumor growth and antiangiogenic treatment effect. Neurosurgery 58: 365-372, 2006.

14. Nguyen DX, Chiang AC, Zhang XH, et al: WNT/TCF signaling through LEF1 and HOXB9 mediates lung adenocarcinoma metastasis. Cell 138: 51-62, 2009.

15. Bobo RH, Laske DW, Akbasak A, Morrison PF, Dedrick RL and Oldfield EH: Convection-enhanced delivery of macromolecules in the brain. Proc Natl Acad Sci USA 91: 2076-2080, 1994.

16. Sampson JH, Brady ML, Petry NA, et al: Intracerebral infusate distribution by convection-enhanced delivery in humans with malignant gliomas: Descriptive effects of target anatomy and catheter positioning. Neurosurgery 60: ONS89-ONS99, 2007.

17. Mamot C, Nguyen JB, Pourdehnad M, et al: Extensive distribution of liposomes in rodent brains and brain tumors following convection-enhanced delivery. J Neurooncol 68: 1-9, 2004.

18. Saini M, Roser F, Samii M and Bellinzona M: A model for intratumoural chemotherapy in the rat brain. Acta Neurochir (Wien) 146: 731-734, 2004.

19. Olson JJ, Zhang Z, Dillehay D and Stubbs J: Assessment of a balloon-tipped catheter modified for intracerebral convection-enhanced delivery. J Neurooncol 89: 159-168, 2008.

20. Morrison PF, Chen MY, Chadwick RS, Lonser RR and Oldfield EH: Focal delivery during direct infusion to brain: Role of flow rate, catheter diameter, and tissue mechanics. Am J Physiol 277: R1218-R1229, 1999.

21. Griffitt W, Glick RP, Lichtor T, Haughton DE and Cohen EP: Development of a new mouse brain tumor model using implantable micro-cannulas. J Neurooncol 41: 117-120, 1999.

22. Morreale VM, Herman BH, Der-Minassian V, et al: A brain-tumor model utilizing stereotactic implantation of a permanent cannula. J Neurosurg 78: 959-965, 1993.

23. Ray P, Wu AM and Gambhir SS: Optical bioluminescence and positron emission tomography imaging of a novel fusion reporter gene in tumor xenografts of living mice. Cancer Res 63: 1160-1165, 2003.

24. Dickinson PJ, LeCouteur RA, Higgins RJ, et al: Canine model of convection-enhanced delivery of liposomes containing CPT-11 monitored with real-time magnetic resonance imaging: Laboratory investigation. J Neurosurg 108: 989-998, 2008.

25. Shah K, Bureau E, Kim DE, et al: Glioma therapy and real-time imaging of neural precursor cell migration and tumor regression. Ann Neurol 57: 34-41, 2005. 